\section{Comment on: The association between the timing of energy intake and the risk of overweight and obesity among Saudi female university students}

\section{To the Editor}

We read with interest the article titled "The association between the timing of energy intake and the risk of overweight and obesity among Saudi female university students". We want to congratulate the author for a significant contribution to the body of scientific literature. The study only included female university students, justified by quoting an increased prevalence of obesity among females in Saudi Arabia. The design of the study aims to explore the relationship between the timing of energy intake and obesity. The author used the word "timing of energy intake" instead of writing "timing of food intake". Although, not everything we consume is converted into energy such as dietary fibers, vitamins, minerals, and digestive adjuvant. The most important finding of the study was that the time of caloric consumption between the 2 groups of female students influenced the development of obesity even though the number of calories consumed was equal. Although the study is complete and plausible, there are few methodological concerns in the article.

First, during menstruation female students may feel hungry and consume more food or may avoid food due to symptoms like abdominal cramps, nausea, and vomiting. ${ }^{2}$ Therefore monitoring food intake in those days will not give us the actual food consumed. Second, the development of obesity is multifactorial, consumption of fast food, changing food preferences, irregular meal time, bulk loading, and disturbed circadian rhythm are some of the important factors in the development of obesity, therefore, dietary intake for 4 consecutive days may not be sufficient to get the actual food habits among female students. Third, the author had mentioned in the study limitations that the level of activity among female participants was not recorded, however, we want to emphasize that the level of activity plays a major role in the development of obesity. Future studies may use gadgets like a smartwatch that would record essential day-to-day mobility data. Fifth, students suffer from a variety of stress during their academic years leading to anxiety, depression as well as eating disorders, which may lead to obesity such as Night eating syndrome. ${ }^{3,4}$ Therefore, the internal validity of the study can be improved by including assessment tools to differentiate students with such disorders.

In conclusion, obesity is a global menace that is accentuated by the consumption of unhealthy fast food and a sedentary lifestyle. This study provides important information on the most current prevalent health problem in Saudi Arabia. Nowadays, Students may not be able to spare time to perform exercises, especially females, therefore the finding of the study such as consuming calories in the morning and avoiding eating late in the evening would be an easy fix among students to avoid obesity. The study also discovered an important gap in the body of scientific knowledge related to the most suitable time to consume food in the morning and the influence of disturbed circadian rhythm on food preferences among female students.

Faizan Z. Kashoo
Mazen S. Alqahtani
Department of Physical Therapy and
Health Rehabilitation
Raed A. Alharbi
Saleh A. Aloyuni
Public Health Department
College of Applied Medical Sciences
Majmaah University
Riyadh, Kingdom of Saudi Arabia

Reply from the Author

No reply was received from the Author.

\section{References}

1. Alamri ES. The risk of overweight and obesity among Saudi female. Saudi Med J 2019; 40: 1272-1277.

2. Balbi C1, Musone R, Menditto A, Di Prisco L, Cassese E, D'Ajello M, et al. Influence of menstrual factors and dietary habits on menstrual pain in adolescence age. Eur J Obstet Gynecol Reprod Biol 2000; 9: 143-148.

3. Ahmad M, Kashoo FZ, Alqahtani M, Sami W, Rizvi M, Bushra A. Relation between night eating syndrome and academic grades among university students. Turk J Endocrinol Metab 2019; 23 .

4. Naji E, Kashoo FZ, Kashoo M. Prevalence of Obesity and Overweight among Majmaah University Students. Indian J Physiother Occup Ther 2013; 7: 41. 